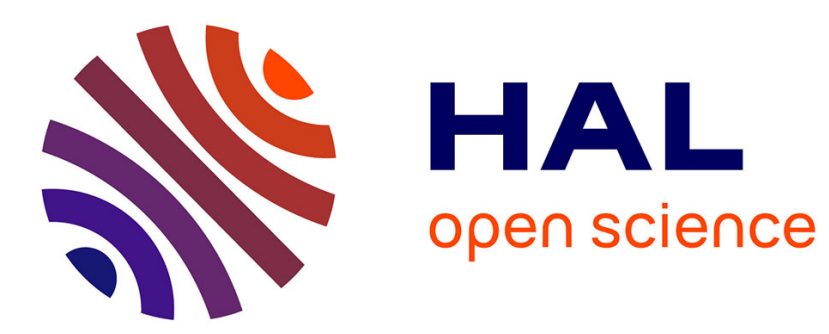

\title{
A new class of identities involving Cauchy numbers, harmonic numbers and zeta values
}

Bernard Candelpergher, Marc-Antoine Coppo

\section{To cite this version:}

Bernard Candelpergher, Marc-Antoine Coppo. A new class of identities involving Cauchy numbers, harmonic numbers and zeta values. Ramanujan Journal (The), 2012, 27, pp.305-328. hal-00495767v9

\section{HAL Id: hal-00495767 \\ https://hal.univ-cotedazur.fr/hal-00495767v9}

Submitted on 19 Dec 2011

HAL is a multi-disciplinary open access archive for the deposit and dissemination of scientific research documents, whether they are published or not. The documents may come from teaching and research institutions in France or abroad, or from public or private research centers.
L'archive ouverte pluridisciplinaire HAL, est destinée au dépôt et à la diffusion de documents scientifiques de niveau recherche, publiés ou non, émanant des établissements d'enseignement et de recherche français ou étrangers, des laboratoires publics ou privés. 


\title{
A new class of identities involving Cauchy numbers, harmonic numbers and zeta values
}

\author{
Bernard Candelpergher and Marc-Antoine Coppo \\ University of Nice Sophia Antipolis \\ Laboratory Jean Alexandre Dieudonné \\ Parc Valrose \\ F-06108 Nice Cedex 2 \\ FRANCE \\ Bernard.CANDELPERGHERQunice.fr \\ Marc-Antoine.COPPO@unice.fr \\ Article to appear in The Ramanujan Journal
}

\begin{abstract}
Improving an old idea of Hermite, we associate to each natural number $k$ a modified zeta function of order $k$. The evaluation of the values of these functions $F_{k}$ at positive integers reveals a wide class of identities linking Cauchy numbers, harmonic numbers and zeta values.
\end{abstract}

Mathematical Subject Classification (2000): 11B83, 11M41, 33B15, 40 G99.

Keywords: Cauchy numbers, Bell polynomials, Harmonic numbers, Laplace-Borel transform, Mellin transform, Zeta values, Ramanujan summation, Hermite's formula.

\section{Introduction}

It has been well known since the second-half of the 19th century that the Riemann zeta function may be represented by the (normalized) Mellin transform (cf. [14])

$$
\zeta(s)=\frac{1}{\Gamma(s)} \int_{0}^{+\infty} t^{s-1} \frac{e^{-t}}{1-e^{-t}} d t \quad \text { for } \Re(s)>1,
$$

and from late works of Hermite (cf. [11]) that one has also

$$
\zeta(s)-\frac{1}{s-1}=\frac{1}{\Gamma(s)} \int_{0}^{+\infty} t^{s-1} \frac{e^{-t}}{1-e^{-t}}\left(\sum_{n=1}^{\infty} \frac{\lambda_{n}}{n !}\left(1-e^{-t}\right)^{n}\right) d t \quad \text { for } \Re(s) \geq 1,
$$


where $\lambda_{1}=\frac{1}{2}$ and $\lambda_{n+1}=\int_{0}^{1} x(1-x) \cdots(n-x) d x$ are the (non-alternating) Cauchy numbers ${ }^{1}$.

Improving Hermite's idea, one may, more generally, consider Mellin transforms of type

$$
F(s)=\frac{1}{\Gamma(s)} \int_{0}^{+\infty} t^{s-1} \frac{e^{-t}}{1-e^{-t}} f\left(1-e^{-t}\right) d t \quad \text { with } f(z)=\sum_{n=1}^{\infty} \omega_{n} \frac{z^{n}}{n^{k}}
$$

for suitable sequences $\left(\omega_{n}\right)_{n \geq 1}$ of rational numbers. The simplest interesting case $\omega_{n}=1$ corresponds to the Arakawa-Kaneko zeta function and has been studied extensively in [8]. In this article, we investigate the case $\omega_{n}=\frac{\lambda_{n}}{n !}$, i.e., we study the function

$F_{k}(s)=\frac{1}{\Gamma(s)} \int_{0}^{+\infty} t^{s-1} \frac{e^{-t}}{1-e^{-t}} f_{k}\left(1-e^{-t}\right) d t \quad$ with $f_{k}(z)=\sum_{n=1}^{\infty} \frac{\lambda_{n}}{n !} \frac{z^{n}}{n^{k}}(k=0,1,2, \ldots)$,

which is a priori defined in the half-plane $\Re(s) \geq 1$ but analytically continues in the whole complex $s$-plane (Theorem 7 ). We call this function $F_{k}$ the modified zeta function of order $k$. An evaluation by two different methods of the values of $F_{k}$ at positive integers $q$ leads to a new class of identities linking Cauchy numbers, harmonic numbers and zeta values. In the case $k=0$, Hermite's formula for $\zeta$ (cf. [7]) is regained, i.e.,

$$
F_{0}(q)=\zeta(q)-\frac{1}{q-1}=\sum_{n=1}^{\infty} \frac{\lambda_{n}}{n ! n} P_{q-1}\left(H_{n}^{(1)}, H_{n}^{(2)}, \ldots, H_{n}^{(q-1)}\right),
$$

where the polynomials $P_{m}$ are the modified Bell polynomials defined by the generating function

$$
\exp \left(\sum_{k=1}^{\infty} x_{k} \frac{z^{k}}{k}\right)=\sum_{m=0}^{\infty} P_{m}\left(x_{1}, \cdots, x_{m}\right) z^{m},
$$

evaluated at harmonic numbers $H_{n}^{(m)}=\sum_{j=1}^{n} \frac{1}{j^{m}}$. In the simplest higher case $k=1$, this extension of Hermite's formula leads to the following new relation (Theorem 10):

$$
\begin{aligned}
F_{1}(q)=\sum_{n=1}^{\infty} & \frac{\lambda_{n}}{n ! n^{2}} P_{q-1}\left(H_{n}, H_{n}^{(2)}, \ldots, H_{n}^{(q-1)}\right)= \\
& \sum_{n=1}^{\infty} \frac{\log (n+1)}{n^{q}}+\gamma \zeta(q)+\zeta(q+1)-\sum_{n=1}^{\infty} \frac{H_{n}}{n^{q}}-\sum_{k=1}^{q-1} \frac{1}{k} \sum_{n=1}^{\infty} \frac{1}{(n+1)^{k} n^{q-k}}
\end{aligned}
$$

where $H_{n}=H_{n}^{(1)}$, and $\gamma=\lim _{n \rightarrow \infty}\left(H_{n}-\log n\right)$ is the Euler-Mascheroni constant.

\footnotetext{
${ }^{1}$ The sequence of numbers $\frac{\lambda_{n}}{n !}$ appeared for the first time in a letter of James Gregory dated back to 1670 (cf. The correspondence of Isaac Newton, vol. 1, p. 46). For this reason, they are sometimes called Gregory coefficients.
} 
For example, for $q=2$, since $P_{1}\left(H_{n}\right)=H_{n}$ and $\sum_{n=1}^{\infty} \frac{H_{n}}{n^{2}}=2 \zeta(3)$ (cf. [6], [7]), then the previous relation may be written

$$
F_{1}(2)=\sum_{n=1}^{\infty} \frac{\lambda_{n} H_{n}}{n ! n^{2}}=\sum_{n=1}^{\infty} \frac{\log (n+1)}{n^{2}}+\gamma \zeta(2)-\zeta(3)-1
$$

and this generalizes the known formula

$$
F_{0}(2)=\sum_{n=1}^{\infty} \frac{\lambda_{n} H_{n}}{n ! n}=\zeta(2)-1
$$

The function $F_{k}$ also has an interesting interpretation in terms of Ramanujan summation (cf. [3]) as underscored by Theorem 11. In particular, one shows the identity

$$
F_{k}(1)=\sum_{n=1}^{\infty} \frac{\lambda_{n}}{n !} \frac{1}{n^{k+1}}=\sum_{n \geq 1}^{\mathcal{R}} \frac{P_{k}\left(H_{n}, H_{n}^{(2)}, \ldots, H_{n}^{(k)}\right)}{n},
$$

where, in the right member, $\sum_{n \geq 1}^{\mathcal{R}}$ denotes the sum (in the sense of Ramanujan) of the divergent series. This raises a kind of reciprocity between $F_{k}(1)$ and $F_{0}(k+1)$.

\section{Preliminaries}

\subsection{The non-alternating Cauchy numbers}

Definition 1. The non-alternating Cauchy numbers (cf. [7], [12]) are the sequence of (positive) rational numbers $\left(\lambda_{n}\right)_{n \geq 1}$ defined by the exponential generating function

$$
\frac{z}{\log (1-z)}+1=\sum_{n \geq 1} \frac{\lambda_{n}}{n !} z^{n} .
$$

Dividing by $z$ and setting $z=1-e^{-t}$ and $t>0$, this relation may be rewritten

$$
\frac{1}{1-e^{-t}}-\frac{1}{t}=\sum_{n=1}^{\infty} \frac{\lambda_{n}}{n !}\left(1-e^{-t}\right)^{n-1}
$$

From (1), one may easily deduce the following recursive relation

$$
\sum_{j=1}^{n} \frac{\lambda_{j}}{j !(n-j+1)}-\frac{1}{n+1}=0 \quad \text { for } n \geq 1 .
$$

Example 1. The first non-alternating Cauchy numbers are

$$
\lambda_{1}=\frac{1}{2}, \lambda_{2}=\frac{1}{6}, \lambda_{3}=\frac{1}{4}, \lambda_{4}=\frac{19}{30}, \lambda_{5}=\frac{9}{4} .
$$




\subsection{The modified Bell polynomials evaluated at harmonic numbers}

Definition 2. The modified Bell polynomials (cf. [5], [7], [10]) are the polynomials $P_{m}$ defined for all natural numbers $m$ by $P_{0}=1$ and the generating function

$$
\exp \left(\sum_{k \geq 1} x_{k} \frac{z^{k}}{k}\right)=1+\sum_{m \geq 1} P_{m}\left(x_{1}, \ldots, x_{m}\right) z^{m} .
$$

The general explicit expression for $P_{m}$ is

$$
P_{m}\left(x_{1}, \ldots, x_{m}\right)=\sum_{k_{1}+2 k_{2}+3 k_{3}+\cdots=m} \frac{1}{k_{1} ! k_{2} ! k_{3} ! \ldots}\left(\frac{x_{1}}{1}\right)^{k_{1}}\left(\frac{x_{2}}{2}\right)^{k_{2}}\left(\frac{x_{3}}{3}\right)^{k_{3}} \ldots
$$

One may also compute recursively the polynomials $P_{m}$ by means of the following relation

$$
m P_{m}\left(x_{1}, \ldots, x_{m}\right)=\sum_{k=1}^{m} x_{k} P_{m-k}\left(x_{1}, \ldots, x_{m-k}\right) \quad(m \geq 1) .
$$

Proposition 1. For all natural numbers $m$, and each integer $n \geq 1$,

$$
\int_{0}^{+\infty} e^{-t}\left(1-e^{-t}\right)^{n-1} \frac{t^{m}}{m !} d t=\frac{P_{m}\left(H_{n}, \ldots, H_{n}^{(m)}\right)}{n},
$$

with

$$
H_{n}^{(m)}=\sum_{j=1}^{n} \frac{1}{j^{m}} \quad \text { and } \quad H_{n}=H_{n}^{(1)} .
$$

Proof. One starts from the classical Euler relation (cf. [14])

$$
\mathrm{B}(a, b)=\int_{0}^{1} u^{a-1}(1-u)^{b-1} d u=\frac{\Gamma(a) \Gamma(b)}{\Gamma(a+b)},
$$

and substitute $u=e^{-t}, a=1-z$, and $b=n+1$; then one obtains

$$
\int_{0}^{+\infty} e^{-t}\left(1-e^{-t}\right)^{n} e^{t z} d t=\frac{n !}{(1-z)(2-z) \ldots(n+1-z)} .
$$

Moreover, one has

$$
\begin{aligned}
\frac{n !}{(1-z)(2-z) \ldots(n+1-z)} & =\frac{n !}{(n+1) !} \times \prod_{j=0}^{n}\left(1-\frac{z}{j+1}\right)^{-1} \\
& =\frac{1}{(n+1)} \times \exp \left(-\sum_{j=0}^{n} \log \left(1-\frac{z}{j+1}\right)\right) \\
& =\frac{1}{(n+1)} \times \exp \left(\sum_{j=0}^{n} \sum_{k=1}^{\infty} \frac{z^{k}}{k(j+1)^{k}}\right) \\
& =\frac{1}{(n+1)} \exp \left(\sum_{k=1}^{\infty} H_{n+1}^{(k)} \frac{z^{k}}{k}\right) \\
& =\sum_{m=0}^{\infty} \frac{P_{m}\left(H_{n+1}^{(1)}, \ldots, H_{n+1}^{(m)}\right)}{n+1} z^{m} \quad(\text { by (3)). }
\end{aligned}
$$


Thus (4) results by identification of the term in $z^{m}$.

Example 2. For small values of $m$, one has

$$
\begin{gathered}
P_{1}\left(H_{n}\right)=H_{n}, P_{2}\left(H_{n}, H_{n}^{(2)}\right)=\frac{\left(H_{n}\right)^{2}}{2}+\frac{H_{n}^{(2)}}{2}, \\
P_{3}\left(H_{n}, H_{n}^{(2)}, H_{n}^{(3)}\right)=\frac{\left(H_{n}\right)^{3}}{6}+\frac{H_{n} H_{n}^{(2)}}{2}+\frac{H_{n}^{(3)}}{3} .
\end{gathered}
$$

\subsection{The Laplace-Borel transformation}

We consider the vector space $E$ of complex-valued functions $f \in \mathcal{C}^{1}(] 0,+\infty[)$ such that

for all $\varepsilon>0$, there exists $C_{\varepsilon}>0$ such that $|f(t)| \leq C_{\varepsilon} e^{\varepsilon t}$ for all $\left.t \in\right] 0,+\infty[$.

In particular, a function $f \in E$ satisfies the following two properties:

a) for all $x$ with $\Re(x)>0, t \mapsto e^{-x t} f(t)$ is integrable on $] 0,+\infty[$,

b) for all $\beta$ with $0<\beta<1, t \mapsto|f(t)| \frac{1}{t^{\beta}}$ is integrable on $] 0,1[$.

We recall now some basic properties (cf. [13]) of the Laplace transformation in this frame which are appropriate for our purpose.

Definition 3. Let $f$ be a function in $E$. The Laplace transform $\mathcal{L}(f)$ of $f$ is defined by

$$
\mathcal{L}(f)(x)=\int_{0}^{+\infty} e^{-x t} f(t) d t \quad \text { for } \Re(x)>0 .
$$

Proposition 2 (cf. [13]). Let $\mathcal{E}=\mathcal{L}(E)$ be the image of $E$ under $\mathcal{L}$. If $a$ is a function in $\mathcal{E}$, then

a) $a$ is an analytic function of $x$ in the half-plane $\Re(x)>0$,

b) $a(x) \rightarrow 0$ when $\Re(x) \rightarrow+\infty$,

c) $\mathcal{L}: E \rightarrow \mathcal{E}$ is an isomorphism.

Definition 4. Let $a \in \mathcal{E}$. The Borel transform of $a$ is the unique function $\widehat{a} \in E$ such that $a=\mathcal{L}(\widehat{a})$. One has the two reciprocal formulas

$$
\widehat{a}(t)=\frac{1}{2 i \pi} \int_{c-i \infty}^{c+i \infty} e^{z t} a(z) d z \quad \text { for all } c>0 \text { and } t>0
$$

and

$$
a(x)=\int_{0}^{+\infty} e^{-x t} \widehat{a}(t) d t \quad \text { for } \Re(x)>0 .
$$


Definition 5. Let $f$ and $g$ be two functions in $E$. The convolution product $f * g$ of $f$ and $g$ is the function defined for all $t>0$ by

$$
(f * g)(t)=\int_{0}^{t} f(u) g(t-u) d u .
$$

Proposition 3 (cf. [13]). If $f \in E$ and $g \in E$, then $f * g \in E$ and

$$
\mathcal{L}(f * g)=\mathcal{L}(f) \mathcal{L}(g) .
$$

Hence, if $a \in \mathcal{E}$ and $b \in \mathcal{E}$ then $a b \in \mathcal{E}$ since $a b=\mathcal{L}(\widehat{a} * \widehat{b})$.

Theorem 1. Let a be a function in $\mathcal{E}$. Then the series

$$
\sum_{n \geq 1} \frac{\lambda_{n}}{n !} \int_{0}^{+\infty} e^{-t}\left(1-e^{-t}\right)^{n-1} \widehat{a}(t) d t
$$

converges and

$$
\sum_{n=1}^{\infty} \frac{\lambda_{n}}{n !} \int_{0}^{+\infty} e^{-t}\left(1-e^{-t}\right)^{n-1} \widehat{a}(t) d t=\int_{0}^{+\infty}\left(\frac{1}{1-e^{-t}}-\frac{1}{t}\right) e^{-t} \widehat{a}(t) d t .
$$

Proof. By (2)

$$
\int_{0}^{+\infty}\left(\frac{1}{1-e^{-t}}-\frac{1}{t}\right) e^{-t} \widehat{a}(t) d t=\int_{0}^{+\infty} \sum_{n=1}^{\infty} \frac{\lambda_{n}}{n !}\left(1-e^{-t}\right)^{n-1} e^{-t} \widehat{a}(t) d t .
$$

In the right member, the order of $\int_{0}^{+\infty}$ and $\sum_{n=1}^{\infty}$ may be interchanged since

$$
\begin{aligned}
\int_{0}^{+\infty} \sum_{n=1}^{\infty}\left|\frac{\lambda_{n}}{n !}\left(1-e^{-t}\right)^{n-1} e^{-t} \widehat{a}(t)\right| d t & =\int_{0}^{+\infty} \sum_{n=1}^{\infty} \frac{\lambda_{n}}{n !}\left(1-e^{-t}\right)^{n-1} e^{-t}|\widehat{a}(t)| d t \\
& =\int_{0}^{+\infty}\left(\frac{1}{1-e^{-t}}-\frac{1}{t}\right) e^{-t}|\widehat{a}(t)| d t
\end{aligned}
$$

and the convergence of this last integral follows from the assumption that $a \in \mathcal{E}$.

Example 3. Let $a(x)=\frac{1}{x^{s}}$ with $\Re(s) \geq 1$. Then $a \in \mathcal{E}$ and $\widehat{a}(t)=\frac{t^{s-1}}{\Gamma(s)}$. Hence

$$
\begin{aligned}
\sum_{n=1}^{\infty} \frac{\lambda_{n}}{n !} \int_{0}^{+\infty} e^{-t}\left(1-e^{-t}\right)^{n-1} \frac{t^{s-1}}{\Gamma(s)} d t & =\frac{1}{\Gamma(s)} \int_{0}^{+\infty} e^{-t}\left(\frac{1}{1-e^{-t}}-\frac{1}{t}\right) t^{s-1} d t \\
& = \begin{cases}\gamma & \text { if } s=1, \\
\zeta(s)-\frac{1}{s-1} & \text { if } s \neq 1\end{cases}
\end{aligned}
$$

where $\gamma$ refers to the Euler constant. In particular, since

$$
\int_{0}^{+\infty} e^{-t}\left(1-e^{-t}\right)^{n-1} d t=\frac{1}{n} \text { for each integer } n \geq 1
$$

then

$$
\gamma=\sum_{n=1}^{\infty} \frac{\lambda_{n}}{n !} \frac{1}{n}
$$




\section{The operator $D$}

Proposition 4. If $a \in \mathcal{E}$, then the integral

$$
\int_{0}^{+\infty} e^{-t}\left(1-e^{-t}\right)^{x-1} \widehat{a}(t) d t
$$

converges for all $x$ with $\Re(x)>0$.

Proof. If $a \in \mathcal{E}$ and $\Re(x)>0$, we may write for $t \in] 0,+\infty[$,

$$
\left|e^{-t}\left(1-e^{-t}\right)^{x-1} \widehat{a}(t)\right| \leq e^{-t} e^{(1-\Re(x))\left(-\log \left(1-e^{-t}\right)\right)}|\widehat{a}(t)| .
$$

The convergence when $t \rightarrow+\infty$ results from the inequality

$$
e^{-t} e^{(1-\Re(x))\left(-\log \left(1-e^{-t}\right)\right)}|\widehat{a}(t)| \leq \frac{e^{-t}}{1-e^{-t}}|\widehat{a}(t)| \leq 2 e^{-t}|\widehat{a}(t)| \quad(\text { for } t \geq \log 2) .
$$

The convergence when $t \rightarrow 0$ results from the inequality

$$
e^{(1-\Re(x))\left(-\log \left(1-e^{-t}\right)\right)} \leq \begin{cases}1 & \text { if } \Re(x) \geq 1, \\ \frac{1}{\left(1-e^{-t}\right)^{(1-\Re(x))}} & \text { if } 0<\Re(x)<1\end{cases}
$$

since the function $t \mapsto e^{-t}|\widehat{a}(t)| \frac{1}{\left(1-e^{-t}\right)^{\beta}}$ is integrable at 0 for $0<\beta<1$ by the definition of $E$ (note that $\left(1-e^{-t}\right)^{-\beta} \leq(k t)^{-1}$ for small enough $t$ ).

Definition 6. Let $a$ be a function in $\mathcal{E}$. We call $D(a)$ the function defined for all $x$ with $\Re(x)>0$ by

$$
D(a)(x)=\int_{0}^{+\infty} e^{-t}\left(1-e^{-t}\right)^{x-1} \widehat{a}(t) d t .
$$

Remark 1. a) By Theorem 1, the series $\sum_{n \geq 1} \frac{\lambda_{n}}{n !} D(a)(n)$ converges and its sum is given by formula (6).

b) The values of $D(a)$ at positive integers may be computed directly without recourse to $\widehat{a}$. The development of $\left(1-e^{-t}\right)^{n}$ by the binomial theorem gives

$$
D(a)(n+1)=\sum_{k=0}^{n}(-1)^{k}\left(\begin{array}{l}
n \\
k
\end{array}\right) a(k+1) \quad \text { for all integer } n \geq 0 .
$$

Definition 7. We call $\Lambda$ the $C^{1}$-diffeomorphism of $\mathbb{R}_{+}$defined by $\Lambda(u)=-\log \left(1-e^{-u}\right)$. In particular, it is important to note that $\Lambda$ is involutive:

$$
\Lambda^{-1}=\Lambda \text {. }
$$


Theorem 2. Let a be a function in $\mathcal{E}$. Then the function $D(a) \in \mathcal{E}$ and, moreover, verifies the relation

$$
\widehat{D(a)}=\widehat{a}(\Lambda)
$$

where $\widehat{a}(\Lambda)$ denotes $\widehat{a} \circ \Lambda$.

Proof. The change of variables $t=\Lambda(u)$ in (7) gives

$$
D(a)(x)=\int_{0}^{+\infty} e^{-x u} \widehat{a}(\Lambda(u)) d u \quad \text { for } \Re(x)>0 .
$$

Thus, $D(a)=\mathcal{L}(\widehat{a}(\Lambda))$. It remains to prove that $D(a) \in \mathcal{E}$. One has only to check that the function $\widehat{a}(\Lambda)$ is in $E$. This function being in $\mathcal{C}^{1}(] 0,+\infty[)$, it suffices to show that for all $\varepsilon>0$, the function $u \mapsto e^{-\varepsilon u}\left|\widehat{a}\left(-\log \left(1-e^{-u}\right)\right)\right|$ is bounded on $] 0,+\infty[$. This results from the existence of $C_{\varepsilon}>0$ such that

$$
\left.\left|\widehat{a}\left(-\log \left(1-e^{-u}\right)\right)\right| \leq C_{\varepsilon}\left(1-e^{-u}\right)^{\varepsilon} \text { for all } u \in\right] 0,+\infty[.
$$

Example 4. Let $a(x)=\frac{1}{x^{s}}$ with $\Re(s) \geq 1$. Then $\widehat{a}(t)=\frac{t^{s-1}}{\Gamma(s)}$. Thus, by (9),

$$
D\left(\frac{1}{x^{s}}\right)=\mathcal{L}\left(\frac{\Lambda^{s-1}}{\Gamma(s)}\right)
$$

and if $s=m+1$ with $m$ a natural number and $n \geq 1$, then by (4),

$$
D\left(\frac{1}{x^{m+1}}\right)(n)=\frac{P_{m}\left(H_{n}, \ldots, H_{n}^{(m)}\right)}{n} .
$$

Remark 2. Theorem 2 may be summarized in the following diagram

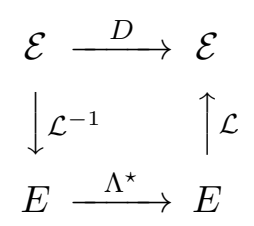

where $\Lambda^{\star}(\widehat{a})=\widehat{a}(\Lambda)$. The algebraic properties of $D$ are summed up in the following theorem.

Theorem 3. The operator $D$ is an automorphism of $\mathcal{E}$ which verifies $D=D^{-1}$ and lets the function $x \mapsto \frac{1}{x}$ invariant.

Proof. We can write $D=\mathcal{L} \Lambda^{\star} \mathcal{L}^{-1}$ and $\Lambda^{\star}$ is an automorphism of $E$ which verifies $\Lambda^{\star}=\left(\Lambda^{\star}\right)^{-1}$ since $\Lambda=\Lambda^{-1}$. Furthermore,

$$
D\left(\frac{1}{x}\right)=\mathcal{L}(1)=\frac{1}{x} .
$$




\section{The harmonic product}

Our aim is to define the harmonic product of two functions $a$ and $b$ in $\mathcal{E}$ as being the unique function $f$ of $\mathcal{E}$ such that

$$
D(a)(x) \cdot D(b)(x)=D(f)(x) .
$$

Thus, we have to establish that such a function exists and is unique. In order to do this, we introduce first a $\Lambda$-convolution product of two functions in $E$.

\subsection{The $\Lambda$-convolution product}

Proposition 5. If $a$ and $b$ are in $\mathcal{E}$, then $\widehat{a}(\Lambda) * \widehat{b}(\Lambda) \in E$.

Proof. From the definition of the convolution product, one may write

$$
\left(\widehat{a}(\Lambda) *(\widehat{b}(\Lambda))(t)=\int_{0}^{t} \widehat{a}(\Lambda(u)) \widehat{b}(\Lambda(t-u)) d u .\right.
$$

Now, for all $\varepsilon>0$, there exists $C_{\varepsilon}>0$ and $D_{\varepsilon}>0$ such that

$$
\begin{aligned}
\left|\widehat{a}\left(-\log \left(1-e^{-u}\right)\right)\right| & \leq C_{\varepsilon}\left(1-e^{-u}\right)^{\varepsilon} \text { and } \\
\left|\widehat{b}\left(-\log \left(1-e^{-(t-u)}\right)\right)\right| & \left.\leq D_{\varepsilon}\left(1-e^{-(t-u)}\right)^{\varepsilon} \text { for all } u \in\right] 0,+\infty[.
\end{aligned}
$$

It follows that

$$
|(\widehat{a}(\Lambda) * \widehat{b}(\Lambda))(t)| \leq C_{\varepsilon} D_{\varepsilon} \int_{0}^{t}\left(1-e^{-u}\right)^{\varepsilon}\left(1-e^{-(t-u)}\right)^{\varepsilon} d u .
$$

One has also

$$
\begin{array}{r}
\int_{0}^{t}\left(1-e^{-u}\right)^{\varepsilon}\left(1-e^{-(t-u)}\right)^{\varepsilon} d u=\left(1-e^{-t}\right)^{1+2 \varepsilon} \int_{0}^{1} u^{\varepsilon}(1-u)^{\varepsilon} \frac{1}{\left(1-\left(1-e^{-t}\right) u\right)^{\varepsilon+1}} d u \\
\leq\left(1-e^{-t}\right)^{1+2 \varepsilon} \int_{0}^{1} \frac{1}{\left(1-\left(1-e^{-t}\right) u\right)^{\varepsilon+1}} d u \leq\left(1-e^{-t}\right)^{1+2 \varepsilon} \frac{e^{t \varepsilon}-1}{\left(1-e^{-t}\right) \varepsilon} \\
\leq\left(1-e^{-t}\right)^{2 \varepsilon} \frac{e^{t \varepsilon}-1}{\varepsilon} \leq \frac{e^{t \varepsilon}}{\varepsilon} .
\end{array}
$$

Hence, $|(\widehat{a}(\Lambda) * \widehat{b}(\Lambda))(t)| \leq C_{\varepsilon} D_{\varepsilon} \frac{e^{t \varepsilon}}{\varepsilon}$, which proves that this function belongs to $E$ as required.

Definition 8. Let $a$ and $b$ be two functions in $\mathcal{E}$. The $\Lambda$-convolution product $\widehat{a} \circledast \widehat{b}$ of $\widehat{a}$ and $\widehat{b}$ is defined by

$$
\widehat{a} \circledast \widehat{b}=\Lambda^{\star}\left(\Lambda^{\star}(\widehat{a}) * \Lambda^{\star}(\widehat{b})\right),
$$

or equivalently ( since $\Lambda^{\star}=\left(\Lambda^{\star}\right)^{-1}$ )

$$
(\widehat{a} \circledast \widehat{b})(\Lambda)=\widehat{a}(\Lambda) * \widehat{b}(\Lambda) .
$$

Remark 3. The $\Lambda$-convolution product inherits the algebraic properties of the ordinary convolution product, i.e., bilinearity, commutativity, and associativity. 


\subsection{The harmonic product}

Definition 9. Let $a$ and $b$ two functions in $\mathcal{E}$. The harmonic product $a \bowtie b$ of $a$ and $b$ is defined by

$$
a \bowtie b=\mathcal{L}(\widehat{a} \circledast \widehat{b}) \in \mathcal{E} .
$$

This construction may be summarized in the following diagram:

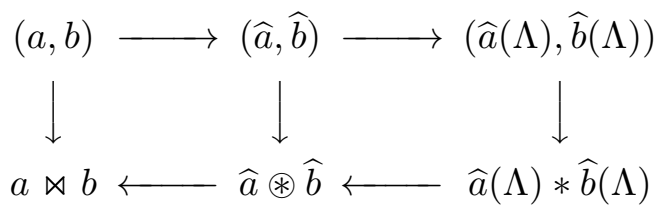

Remark 4. The harmonic product inherits the properties of the $\Lambda$-convolution product: it is bilinear, commutative and associative.

Theorem 4. Let $a$ and $b$ be in $\mathcal{E}$. Then,

$$
D(a \bowtie b)=D(a) D(b),
$$

and

$$
D(a b)=D(a) \bowtie D(b) .
$$

Proof. One knows from Theorem 2 that

$$
D=\mathcal{L} \Lambda^{\star} \mathcal{L}^{-1} .
$$

Hence

$$
D(a \bowtie b)=\mathcal{L} \Lambda^{\star} \mathcal{L}^{-1}(a \bowtie b)=\mathcal{L} \Lambda^{\star}(\widehat{a} \circledast \widehat{b})=\mathcal{L}\left(\Lambda^{\star}(\widehat{a}) * \Lambda^{\star}(\widehat{b})\right),
$$

and it follows from (5) and (9) that

$$
\mathcal{L}\left(\Lambda^{\star}(\widehat{a}) * \Lambda^{\star}(\widehat{b})\right)=\mathcal{L}\left(\Lambda^{\star}(\widehat{a})\right) \mathcal{L}\left(\Lambda^{\star}(\widehat{b})\right)=D(a) D(b)
$$

which proves (12). Moreover, (12) enables us to write

$$
D(D(a) \bowtie D(b))=D^{2}(a) D^{2}(b)=a b \quad\left(\text { since } D=D^{-1}\right),
$$

and so

$$
D(a b)=D^{2}(D(a) \bowtie D(b))=D(a) \bowtie D(b)
$$

which proves (13).

Remark 5. The values of $(a \bowtie b)(n)$ may be computed without recourse to $\widehat{a}$ and $\widehat{b}$. By elementary transformations, it can be shown that

$$
(a \bowtie b)(n+1)=\int_{0}^{+\infty} \int_{0}^{+\infty}\left(e^{-t-s}\right)\left(e^{-t}+e^{-s}-e^{-t} e^{-s}\right)^{n} \widehat{a}(t) \widehat{b}(s) d t d s .
$$


Hence, if the numbers $C_{n}^{k, l}$ are defined by

$$
(X+Y-X Y)^{n}=\sum_{\substack{0 \leq k \leq n \\ 0 \leq l \leq n}} C_{n}^{k, l} X^{k} Y^{l},
$$

then one has the following explicit formula:

$$
(a \bowtie b)(n+1)=\sum_{\substack{0 \leq k \leq n \\ 0 \leq l \leq n}} C_{n}^{k, l} a(k+1) b(l+1),
$$

which can be rewritten in the following equivalent form:

$$
(a \bowtie b)(n+1)=\sum_{0 \leq l \leq k \leq n}(-1)^{k-l}\left(\begin{array}{l}
n \\
k
\end{array}\right)\left(\begin{array}{l}
k \\
l
\end{array}\right) a(k+1) b(n+1-l) \quad(n \geq 0) .
$$

For small values of $n$, this enables one to compute

$$
\begin{aligned}
& (a \bowtie b)(1)=a(1) b(1), \\
& (a \bowtie b)(2)=a(2) b(1)+a(1) b(2)-a(2) b(2), \\
& (a \bowtie b)(3)=a(3) b(1)+a(1) b(3)+2 a(2) b(2)-2 a(3) b(2)-2 a(2) b(3)+a(3) b(3) .
\end{aligned}
$$

Theorem 5. Let

$$
\left(\frac{1}{x}\right)^{\bowtie k}=\underbrace{\frac{1}{x} \bowtie \frac{1}{x} \bowtie \cdots \bowtie \frac{1}{x}}_{k} \quad(k=1,2,3, \cdots),
$$

where $\frac{1}{x}$ denotes (improperly) the function $x \mapsto \frac{1}{x}$. Then, for all natural numbers $m \geq 0$,

$$
\left(\frac{1}{x}\right)^{\bowtie(m+1)}=D\left(\frac{1}{x^{m+1}}\right)
$$

In particular, for all integers $n \geq 1$,

$$
\left(\frac{1}{x}\right)^{\bowtie(m+1)}(n)=\frac{P_{m}\left(H_{n}, \ldots, H_{n}^{(m)}\right)}{n} .
$$

Proof. By (13) we have

$$
D\left(\frac{1}{x^{m+1}}\right)=D(\underbrace{\frac{1}{x} \ldots \frac{1}{x}}_{m+1})=\left(D\left(\frac{1}{x}\right)\right)^{\bowtie(m+1)}=\left(\frac{1}{x}\right)^{\bowtie(m+1)} \text { since } D\left(\frac{1}{x}\right)=\frac{1}{x} .
$$

Thus, (14) results from (11). 


\subsection{The harmonic property}

The following theorem explains the main reason why the harmonic product is called 'harmonic'.

Theorem 6. Let $a \in \mathcal{E}$. Then

$$
\frac{1}{x} \bowtie a=\frac{A(x)}{x},
$$

where $A$ denotes the function defined for $\Re(x)>0$ by

$$
A(x)=\int_{0}^{+\infty} \frac{e^{-x t}-1}{e^{-t}-1} e^{-t} \widehat{a}(t) d t .
$$

In particular, for each integer $n \geq 1$,

$$
\left(\frac{1}{x} \bowtie a\right)(n)=\frac{A(n)}{n}=\frac{1}{n}\left(\sum_{k=1}^{n} a(k)\right) .
$$

Proof. By the definition of the harmonic product, one has

$$
\frac{1}{x} \bowtie a=\mathcal{L}(1 \circledast \widehat{a}) .
$$

Now

$$
(1 \circledast \widehat{a})(\Lambda(u))=(1 * \widehat{a}(\Lambda))(u)=\int_{0}^{u} \widehat{a}(\Lambda(v)) d v=-\int_{+\infty}^{\Lambda(u)} \widehat{a}(t) \frac{e^{-t}}{1-e^{-t}} d t
$$

(by the change of variables $t=\Lambda(v)$ ). Hence,

$$
(1 \circledast \widehat{a})(u)=\int_{u}^{+\infty} \widehat{a}(t) \frac{e^{-t}}{1-e^{-t}} d t .
$$

Thus, we have

$$
\begin{aligned}
\frac{1}{x} \bowtie a & =\int_{0}^{+\infty} e^{-x u}\left(\int_{u}^{+\infty} \widehat{a}(t) \frac{e^{-t}}{1-e^{-t}} d t\right) d u \\
& =\int_{0}^{+\infty}\left(\int_{0}^{t} e^{-x u} d u\right) \widehat{a}(t) \frac{e^{-t}}{1-e^{-t}} d t \\
& =\frac{1}{x} \int_{0}^{+\infty}\left(1-e^{-x t}\right) \widehat{a}(t) \frac{e^{-t}}{1-e^{-t}} d t \\
& =\frac{A(x)}{x} .
\end{aligned}
$$

Furthermore, for each integer $n \geq 1$, we have

$$
A(n)=\int_{0}^{+\infty} \frac{e^{-n t}-1}{e^{-t}-1} e^{-t} \widehat{a}(t) d t=\sum_{k=1}^{n} a(k) .
$$




\section{Example 5.}

$$
\frac{1}{x} \bowtie \frac{1}{x}=D\left(\frac{1}{x^{2}}\right)=\mathcal{L}(\Lambda)=\frac{H(x)}{x} \quad \text { with } H(x)=\psi(x+1)+\gamma,
$$

$\psi$ denoting the logarithmic derivative of $\Gamma$. In particular, for each integer $n \geq 1$,

$$
\left(\frac{1}{x} \bowtie \frac{1}{x}\right)(n)=\frac{H(n)}{n}=\frac{H_{n}}{n} .
$$

Example 6. For $\Re(s) \geq 1$,

$$
\frac{1}{x} \bowtie \frac{1}{x^{s}}=\frac{H^{(s)}(x)}{x},
$$

with

$$
H^{(s)}(x)=\frac{1}{\Gamma(s)} \int_{0}^{+\infty} \frac{1-e^{-x t}}{1-e^{-t}} e^{-t} t^{s-1} d t .
$$

For each integer $n \geq 1$,

$$
\left(\frac{1}{x} \bowtie \frac{1}{x^{s}}\right)(n)=\frac{H^{(s)}(n)}{n}=\frac{H_{n}^{(s)}}{n}=\frac{1}{n}\left(\sum_{m=1}^{n} \frac{1}{m^{s}}\right) .
$$

From (15), by induction on $k$, we deduce the following important corollary.

Corollary 1. For each integer $k \geq 2$,

$$
\left(\left(\frac{1}{x}\right)^{\bowtie k} \bowtie a\right)(n)=\frac{1}{n}\left(\sum_{n \geq n_{1} \geq \cdots \geq n_{k} \geq 1} \frac{a\left(n_{k}\right)}{n_{1} \ldots n_{k-1}}\right) .
$$

Example 7. Applying (16) with $a(x)=\frac{1}{x}$ (and $k=m$ ), we get

$$
\left(\frac{1}{x}\right)^{\bowtie(m+1)}(n)=\frac{1}{n}\left(\sum_{n \geq n_{1} \geq \cdots \geq n_{m} \geq 1} \frac{1}{n_{1} \ldots n_{m}}\right) .
$$

Hence, it follows from (14) and (17) that

$$
P_{m}\left(H_{n}, H_{n}^{(2)}, \ldots, H_{n}^{(m)}\right)=\sum_{n \geq n_{1} \geq \cdots \geq n_{m} \geq 1} \frac{1}{n_{1} \ldots n_{m}},
$$

which is a nice reformulation of Dilcher's formula (cf. [2], [9]).

\section{The modified zeta function $F_{k}$}

\subsection{Integral representation}

Definition 10. For all $s \in \mathbb{C}$ with $\Re(s) \geq 1$ and each natural number $k$, the modified zeta function of order $k$ is defined by

$$
F_{k}(s)=\frac{1}{\Gamma(s)} \int_{0}^{+\infty} t^{s-1} \frac{e^{-t}}{1-e^{-t}} f_{k}\left(1-e^{-t}\right) d t \quad \text { with } \quad f_{k}(z)=\sum_{n=1}^{\infty} \frac{\lambda_{n}}{n !} \frac{z^{n}}{n^{k}} .
$$


Remark 6. By (2) and Example 3, one has $F_{0}(s)=\zeta(s)-\frac{1}{s-1}$.

The fact that $F_{k}$ may be represented by a Mellin transform enables us to analytically continue this function outside its half-plane of definition by a standard analytic method (cf. [14] section 6.7).

Theorem 7. The function $F_{k}$ analytically continues in the whole complex plane as an entire function.

Proof. The function $z \mapsto \frac{1}{\log (1-z)}+\frac{1}{z}$ being analytic in the disc $D(0,1)$ with a singularity at 1 , we deduce from (1) that the radius of convergence of the series $\sum_{n=1}^{\infty} \frac{\lambda_{n} z^{n}}{n !}$ is equal to 1 . Thus 1 is also the radius of convergence of the series $\sum_{n=1}^{\infty} \frac{\lambda_{n} z^{n}}{n ! n^{k}}$ which defines an analytic function $f_{k}$ in the disc $D(0,1)$. Hence, the function

$$
g_{k}: t \mapsto f_{k}\left(1-e^{-t}\right)
$$

is analytic for all $t \in \mathbb{C}$ such that $1-e^{-t} \in D(0,1)$. Since $1-e^{0}=0$, it follows that $g_{k}$ is analytic in a neighbourhood of 0 . Since $g_{k}(0)=0$, the function $t \mapsto g_{k}(t) \frac{e^{-t}}{1-e^{-t}}$ is itself analytic in a neighbourhood of 0 . It follows that its Mellin transform analytically continues in the complex plane with simple poles at negative integers which are all cancelled by the poles of $\Gamma$.

Theorem 8. For all $s$ with $\Re(s)>1$ and each integer $k \geq 1$,

$F_{k}(s)=\vartheta(k) \zeta(s)+\sum_{j=1}^{k}(-1)^{j} \vartheta(k-j) Z_{j}(s)+(-1)^{k} \frac{1}{\Gamma(s)} \int_{0}^{+\infty} t^{s-1} \frac{e^{-t}}{1-e^{-t}} T^{k}\left(\frac{e^{-t}-1}{t}\right) d t$

with

$$
\begin{aligned}
\vartheta(k) & =\sum_{n=1}^{\infty} \frac{\lambda_{n}}{n !} \frac{1}{n^{k}}, \\
Z_{j}(s) & =\sum_{n>n_{1}>n_{2}>\cdots>n_{j}>0} \frac{1}{n^{s} n_{1} n_{2} \ldots n_{j}}, \\
T f(t) & =\int_{t}^{+\infty} \frac{e^{-u}}{1-e^{-u}} f(u) d u .
\end{aligned}
$$

Proof. Formula (20) results from the integral representation (19) and the two following lemmas.

Lemma 1. For all $t>0$,

$$
f_{k}\left(1-e^{-t}\right)=\sum_{j=0}^{k}(-1)^{j} \vartheta(k-j) \frac{\Lambda^{j}(t)}{j !}+(-1)^{k} T^{k}\left(\frac{e^{-t}-1}{t}\right),
$$

where $\vartheta$ is defined by (21) and $T$ is the operator defined by (23). 
Proof. Let $g_{k}(t)=f_{k}\left(1-e^{-t}\right)$. The function $g_{k}$ verifies the recursive relation

$$
g_{k}^{\prime}(t)=e^{-t} f_{k}^{\prime}\left(1-e^{-t}\right)=\frac{e^{-t}}{1-e^{-t}} f_{k-1}\left(1-e^{-t}\right)=\frac{e^{-t}}{1-e^{-t}} g_{k-1}(t) .
$$

Thus

$$
g_{k}(t)=\int_{0}^{t} \frac{e^{-u}}{1-e^{-u}} g_{k-1}(u) d u=g_{k}(+\infty)-\int_{t}^{+\infty} \frac{e^{-u}}{1-e^{-u}} g_{k-1}(u) d u
$$

with

$$
g_{k}(+\infty)=f_{k}(1)=\vartheta(k)
$$

Thus, one has

$$
g_{k}(t)=\vartheta(k)-\int_{t}^{+\infty} \frac{e^{-u}}{1-e^{-u}} g_{k-1}(u) d u=\vartheta(k)-T\left(g_{k-1}\right),
$$

and a repeated iteration $k$ times of this relation gives

$$
g_{k}(t)=\sum_{j=0}^{k-1} \vartheta(k-j)(-1)^{j} T^{j}(1)+(-1)^{k} T^{k}\left(g_{0}\right) .
$$

Now, by (2),

$$
g_{0}(t)=\sum_{n=1}^{\infty} \frac{\lambda_{n}\left(1-e^{-t}\right)^{n}}{n !}=\frac{e^{-t}-1}{t}+1
$$

and thus

$$
T^{k}\left(g_{0}\right)=T^{k}\left(\frac{e^{-t}-1}{t}\right)+T^{k}(1)
$$

Hence

$$
g_{k}(t)=\sum_{j=0}^{k-1} \vartheta(k-j)(-1)^{j} T^{j}(1)+(-1)^{k} T^{k}(1)+(-1)^{k} T^{k}\left(\frac{e^{-t}-1}{t}\right) .
$$

Since $\vartheta(0)=\sum_{n=1}^{\infty} \frac{\lambda_{n}}{n !}=1$ (by (1) and a tauberian theorem), one deduces that

$$
g_{k}(t)=\sum_{j=0}^{k} \vartheta(k-j)(-1)^{j} T^{j}(1)+(-1)^{k} T^{k}\left(\frac{e^{-t}-1}{t}\right),
$$

and, now, it remains to prove that

$$
\frac{\Lambda^{j}(t)}{j !}=T^{j}(1)
$$

which follows from the recursive relation

$$
\frac{\Lambda^{j}(t)}{j !}=-\int_{+\infty}^{t} \frac{e^{-u}}{1-e^{-u}} \frac{\Lambda^{j-1}(u)}{(j-1) !} d u=T\left(\frac{\Lambda^{j-1}}{(j-1) !}\right) .
$$


Lemma 2. Let $Z_{j}(s)$ be defined by (22). Then, for all $s \in \mathbb{C}$ with $\Re(s)>1$,

$$
Z_{j}(s)=\frac{1}{\Gamma(s)} \int_{0}^{+\infty} t^{s-1} \frac{e^{-t}}{1-e^{-t}} \frac{\Lambda^{j}(t)}{j !} d t .
$$

Proof. From the recursive relation

$$
\partial \frac{\Lambda^{j}(t)}{j !}=\frac{\Lambda^{j-1}(t)}{(j-1) !} \partial \Lambda(t)=-\frac{e^{-t}}{1-e^{-t}} \frac{\Lambda^{j-1}(t)}{(j-1) !}=-\sum_{m>0} e^{-m t} \frac{\Lambda^{j-1}(t)}{(j-1) !},
$$

and $\Lambda(t)=\sum_{n>0} \frac{e^{-n t}}{n}$, one may check by induction on $j$ that

$$
\frac{\Lambda^{j}(t)}{j !}=\sum_{n_{1}>n_{2}>\ldots>n_{j}>0} \frac{e^{-n_{1} t}}{n_{1}} \frac{1}{n_{2}} \cdots \frac{1}{n_{j}} .
$$

Furthermore, one has

$$
\frac{1}{\Gamma(s)} \int_{0}^{+\infty} t^{s-1} e^{-N t} \frac{e^{-t}}{1-e^{-t}} d t=\sum_{n>N} \frac{1}{n^{s}} \quad(\text { for } \Re(s)>1) .
$$

Hence

$$
\frac{1}{\Gamma(s)} \int_{0}^{+\infty} t^{s-1} \frac{e^{-t}}{1-e^{-t}} \frac{\Lambda^{j}(t)}{j !} d t=\sum_{n>n_{1}>n_{2}>\cdots>n_{j}>0} \frac{1}{n^{s}} \frac{1}{n_{1}} \frac{1}{n_{2}} \cdots \frac{1}{n_{j}}=Z_{j}(s) .
$$

\subsection{Values of $F_{k}$ at integers}

Theorem 9. For all $s$ in $\mathbb{C}$ with $\Re(s) \geq 1$ and each natural number $k$, then

$$
F_{k}(s)=\sum_{n=1}^{\infty} \frac{\lambda_{n}}{n ! n^{k}} D\left(\frac{1}{x^{s}}\right)(n) .
$$

In particular, for all natural numbers $m$,

$$
F_{k}(m+1)=\sum_{n=1}^{\infty} \frac{\lambda_{n}}{n !} \frac{P_{m}\left(H_{n}, H_{n}^{(2)}, \ldots, H_{n}^{(m)}\right)}{n^{k+1}} .
$$

Proof. The change of variables $t=\Lambda(u)$ in (19) enables to write

$$
F_{k}(s)=\frac{1}{\Gamma(s)} \int_{0}^{+\infty} f_{k}\left(e^{-u}\right)(\Lambda(u))^{s-1} d u .
$$

Since $D\left(\frac{1}{x^{s}}\right)=\mathcal{L}\left(\frac{\Lambda^{s-1}}{\Gamma(s)}\right)$, we deduce (24) from this last expression of $F_{k}(s)$. Moreover, by (11), one also has $D\left(\frac{1}{x^{m+1}}\right)(n)=\frac{P_{m}\left(H_{n}, \ldots, H_{n}^{(m)}\right)}{n}$, which proves $(25)$. 
Corollary 2. Let $\vartheta(s)$ be the Dirichlet series defined for $\Re(s)>0$ by

$$
\vartheta(s)=\sum_{n=1}^{\infty} \frac{\lambda_{n}}{n !} \frac{1}{n^{s}}
$$

Then for each natural number $k$,

$$
\vartheta(k+1)=F_{k}(1)
$$

\section{Example 8.}

$$
\begin{aligned}
& F_{0}(1)=\sum_{n=1}^{\infty} \frac{\lambda_{n}}{n ! n}=\gamma=\vartheta(1), \\
& F_{0}(2)=\sum_{n=1}^{\infty} \frac{\lambda_{n} H_{n}}{n ! n}=\zeta(2)-1, \\
& F_{0}(3)=\frac{1}{2} \sum_{n=1}^{\infty} \frac{\lambda_{n} H_{n}^{2}}{n ! n}+\frac{1}{2} \sum_{n=1}^{\infty} \frac{\lambda_{n} H_{n}^{(2)}}{n ! n}=\zeta(3)-\frac{1}{2}, \\
& F_{1}(1)=\sum_{n=1}^{\infty} \frac{\lambda_{n}}{n ! n^{2}}=\vartheta(2), \\
& F_{1}(2)=\sum_{n=1}^{\infty} \frac{\lambda_{n} H_{n}}{n ! n^{2}}, \\
& F_{1}(3)=\frac{1}{2} \sum_{n=1}^{\infty} \frac{\lambda_{n} H_{n}^{2}}{n ! n^{2}}+\frac{1}{2} \sum_{n=1}^{\infty} \frac{\lambda_{n} H_{n}^{(2)}}{n ! n^{2}} .
\end{aligned}
$$

5.3 Identities linking Cauchy numbers, harmonic numbers and zeta values

Theorem 10. For all integers $q \geq 2$,

$$
\begin{aligned}
F_{1}(q)= & \sum_{n=1}^{\infty} \frac{\lambda_{n}}{n ! n^{2}} P_{q-1}\left(H_{n}, H_{n}^{(2)}, \ldots, H_{n}^{(q-1)}\right)= \\
& \sum_{n=1}^{\infty} \frac{\log (n+1)}{n^{q}}+\gamma \zeta(q)+\zeta(q+1)-\sum_{n=1}^{\infty} \frac{H_{n}}{n^{q}}-\sum_{k=1}^{q-1} \frac{1}{k} \sum_{n=1}^{\infty} \frac{1}{(n+1)^{k} n^{q-k}} .
\end{aligned}
$$

Proof. By (20) and (25), one may write

$$
\begin{aligned}
& F_{k}(q)=\sum_{n=1}^{\infty} \frac{\lambda_{n}}{n ! n^{k+1}} P_{q-1}\left(H_{n}, H_{n}^{(2)}, \ldots, H_{n}^{(q-1)}\right)= \\
& \vartheta(k) \zeta(q)+\sum_{j=1}^{k}(-1)^{j} \vartheta(k-j) Z_{j}(q)+(-1)^{k} \frac{1}{\Gamma(q)} \int_{0}^{+\infty} t^{q-1} \frac{e^{-t}}{1-e^{-t}} T^{k}\left(\frac{e^{-t}-1}{t}\right) d t .
\end{aligned}
$$


We apply now (28) with $k=1$. This gives

$$
F_{1}(q)=\gamma \zeta(q)-\sum_{n \geq 1} \frac{H_{n-1}}{n^{q}}+\frac{1}{\Gamma(q)} \int_{0}^{+\infty} t^{q-1} \frac{e^{-t}}{1-e^{-t}} \mathrm{E}_{1}(t) d t
$$

with $\mathrm{E}_{1}(t)=-\operatorname{Ei}(-t)=\int_{t}^{+\infty} \frac{e^{-u}}{u} d u$. Thus

$$
F_{1}(q)=\gamma \zeta(q)-\sum_{n \geq 1} \frac{H_{n}}{n^{q}}+\zeta(q+1)+I(q)
$$

where

$$
I(q)=\frac{1}{\Gamma(q)} \int_{0}^{+\infty} t^{q-1} \frac{e^{-t}}{1-e^{-t}} \mathrm{E}_{1}(t) d t=\frac{1}{\Gamma(q)} \sum_{n=1}^{\infty} \int_{0}^{+\infty} e^{-n t} t^{q-1} \mathrm{E}_{1}(t) d t .
$$

Since

$$
\mathrm{E}_{1}(t)=-\gamma-\log t+\sum_{n=1}^{\infty} \frac{(-1)^{n-1}}{n} \frac{t^{n}}{n !}
$$

and $-\gamma-\log t=\frac{\widehat{\log x}}{x}$ (cf. [13]), then $\mathrm{E}_{1}=\frac{\widehat{\log (x+1)}}{x}$. Thus

$$
\int_{0}^{+\infty} e^{-n t} t^{q-1} \mathrm{E}_{1}(t) d t=(-1)^{q-1}\left(\frac{\log (x+1)}{x}\right)^{(q-1)}(n) .
$$

Hence, by a calculation of the $(q-1)$ th derivative, we get

$$
I(q)=\frac{(-1)^{q-1}}{(q-1) !} \sum_{n=1}^{\infty}\left(\frac{\log (x+1)}{x}\right)^{(q-1)}(n)=\sum_{n=1}^{\infty} \frac{\log (n+1)}{n^{q}}-\sum_{k=1}^{q-1} \frac{1}{k} \sum_{n=1}^{\infty} \frac{1}{(n+1)^{k} n^{q-k}} .
$$

Remark 7. 1) We recall Euler's formula (cf. [6])

$$
\sum_{n=1}^{\infty} \frac{H_{n}}{n^{q}}= \begin{cases}\frac{1}{2}(q+2) \zeta(q+1)-\frac{1}{2} \sum_{k=1}^{q-2} \zeta(k+1) \zeta(q-k) & \text { for } q>2 \\ 2 \zeta(3) & \text { for } q=2\end{cases}
$$

2) From $\sum_{n=1}^{\infty} \frac{1}{(n+1) n}=1$, and the decomposition

$$
\frac{1}{(n+1)^{k} n^{q-k}}=\frac{1}{(n+1)^{k-1} n^{q-k}}-\frac{1}{(n+1)^{k} n^{q-k-1}} \quad(0<k<q),
$$

the sum of the series $\sum_{n=1}^{\infty} \frac{1}{(n+1)^{k} n^{q-k}}$ may be expressed as a linear combination of zeta values and integers. 


\section{Example 9.}

$$
\begin{aligned}
& \sum_{n=1}^{\infty} \frac{\log (n+1)}{n^{2}}+\gamma \zeta(2)-\zeta(3)-1=\sum_{n=1}^{\infty} \frac{\lambda_{n} H_{n}}{n ! n^{2}} \\
& \sum_{n=1}^{\infty} \frac{\log (n+1)}{n^{3}}+\gamma \zeta(3)-\frac{1}{10} \zeta(2)^{2}-\frac{1}{2} \zeta(2)=\frac{1}{2} \sum_{n=1}^{\infty} \frac{\lambda_{n} H_{n}^{2}}{n ! n^{2}}+\frac{1}{2} \sum_{n=1}^{\infty} \frac{\lambda_{n} H_{n}^{(2)}}{n ! n^{2}} \\
& \sum_{n=1}^{\infty} \frac{\log (n+1)}{n^{4}}+\gamma \zeta(4)-2 \zeta(5)+\zeta(2) \zeta(3)-\frac{2}{3} \zeta(3)+\frac{1}{3} \zeta(2)-\frac{1}{2}= \\
& \frac{1}{6} \sum_{n=1}^{\infty} \frac{\lambda_{n} H_{n}^{3}}{n ! n^{2}}+\frac{1}{2} \sum_{n=1}^{\infty} \frac{\lambda_{n} H_{n} H_{n}^{(2)}}{n ! n^{2}}+\frac{1}{3} \sum_{n=1}^{\infty} \frac{\lambda_{n} H_{n}^{(3)}}{n ! n^{2}}
\end{aligned}
$$

\subsection{Link with the Ramanujan summation}

The function $F_{k}$ has strong connections with the Ramanujan summation (cf. [3], [4]).

If $a \in \mathcal{E}$, then the series $\sum_{n \geq 1} a(n)$ may be written

$$
\sum_{n \geq 1} a(n)=\sum_{n \geq 1} \int_{0}^{+\infty} e^{-n t} \widehat{a}(t) d t
$$

and a formal permutation of $\sum_{n \geq 1}$ and $\int_{0}^{+\infty}$ would lead us to write

$$
\sum_{n \geq 1} a(n)=\int_{0}^{+\infty} \frac{1}{1-e^{-t}} e^{-t} \widehat{a}(t) d t
$$

However, this last integral may be divergent at 0 . Nevertheless we can renormalize it by removing the singularity at zero. This may be done merely by subtracting the polar part $\frac{1}{t}$ of $\frac{1}{1-e^{-t}}$. From Theorem 1, we know that

$$
\int_{0}^{+\infty}\left(\frac{1}{1-e^{-t}}-\frac{1}{t}\right) e^{-t} \widehat{a}(t) d t=\sum_{n=1}^{\infty} \frac{\lambda_{n}}{n !} \int_{0}^{+\infty} e^{-t}\left(1-e^{-t}\right)^{n-1} \widehat{a}(t) d t=\sum_{n=1}^{\infty} \frac{\lambda_{n}}{n !} D(a)(n) .
$$

This justifies the following definition.

Definition 11. Let $a$ be a function in $\mathcal{E}=\mathcal{L}(E)$. The Ramanujan sum of the series $\sum_{n \geq 1} a(n)$ is defined by

$$
\sum_{n \geq 1}^{\mathcal{R}} a(n)=\int_{0}^{+\infty}\left(\frac{1}{1-e^{-t}}-\frac{1}{t}\right) e^{-t} \widehat{a}(t) d t=\sum_{n=1}^{\infty} \frac{\lambda_{n}}{n !} D(a)(n) .
$$

Lemma 3. Let $a$ and $b$ in $\mathcal{E}$. Then

$$
\sum_{n \geq 1}^{\mathcal{R}}(a \bowtie b)(n)=\sum_{n=1}^{\infty} \frac{\lambda_{n}}{n !} D(a)(n) D(b)(n) .
$$


Proof. This results directly from (12) and (29).

Theorem 11. for all $s \in \mathbb{C}$ with $\Re(s) \geq 1$, one has

$$
F_{0}(s)=\sum_{n \geq 1}^{\mathcal{R}} \frac{1}{n^{s}} \quad \text { and } \quad F_{k}(s)=\sum_{n \geq 1}^{\mathcal{R}}\left(\left(\frac{1}{x}\right)^{\bowtie k} \bowtie \frac{1}{x^{s}}\right)(n) \quad \text { for } k \geq 1 .
$$

Proof. By (24) and (30), taking into account the invariance of $\frac{1}{x}$ by $D$, one may write

$$
\begin{aligned}
\sum_{n \geq 1}^{\mathcal{R}}\left(\left(\frac{1}{x}\right)^{\bowtie k} \bowtie \frac{1}{x^{s}}\right)(n) & =\sum_{n=1}^{\infty} \frac{\lambda_{n}}{n !} D\left(\left(\frac{1}{x}\right)^{\bowtie k}\right)(n) D\left(\frac{1}{x^{s}}\right)(n) \\
& =\sum_{n=1}^{\infty} \frac{\lambda_{n}}{n !}\left(\frac{1}{x}\right)^{k}(n) D\left(\frac{1}{x^{s}}\right)(n) \\
& =\sum_{n=1}^{\infty} \frac{\lambda_{n}}{n ! n^{k}} D\left(\frac{1}{x^{s}}\right)(n)=F_{k}(s) .
\end{aligned}
$$

In particular, by (14), one deduces from (31) the following identity.

Corollary 3. For each natural number $k$,

$$
F_{k}(1)=\vartheta(k+1)=\sum_{n=1}^{\infty} \frac{\lambda_{n}}{n !} \frac{1}{n^{k+1}}=\sum_{n \geq 1}^{\mathcal{R}} \frac{P_{k}\left(H_{n}, H_{n}^{(2)}, \ldots, H_{n}^{(k)}\right)}{n} .
$$

\section{Example 10.}

$$
\begin{aligned}
& \vartheta(1)=\sum_{n=1}^{\infty} \frac{\lambda_{n}}{n ! n}=\sum_{n \geq 1}^{\mathcal{R}} \frac{1}{n}=\gamma, \\
& \vartheta(2)=\sum_{n=1}^{\infty} \frac{\lambda_{n}}{n ! n^{2}}=\sum_{n \geq 1}^{\mathcal{R}} \frac{H_{n}}{n}, \\
& \vartheta(3)=\sum_{n=1}^{\infty} \frac{\lambda_{n}}{n ! n^{3}}=\frac{1}{2} \sum_{n \geq 1}^{\mathcal{R}} \frac{H_{n}^{2}}{n}+\frac{1}{2} \sum_{n \geq 1}^{\mathcal{R}} \frac{H_{n}^{(2)}}{n} .
\end{aligned}
$$

Remark 8. Comparing (32) with

$$
F_{0}(k+1)=\sum_{n=1}^{\infty} \frac{\lambda_{n}}{n ! n} P_{k}\left(H_{n}, H_{n}^{(2)}, \ldots, H_{n}^{(k)}\right)
$$

one may observe a kind of reciprocity between $F_{k}(1)$ and $F_{0}(k+1)$. This results from the fact that $D=D^{-1}$. 
Remark 9. In the case $q=1,(27)$ is meaningless since both the series $\sum_{n \geq 1} \frac{\log (n+1)}{n}$ and $\sum_{n \geq 1} \frac{H_{n}}{n}$ diverge. However, since

$$
\log (x+1)-(\psi(x+1)+\gamma)=\int_{0}^{+\infty}\left(e^{-x u}-1\right)\left(\frac{1}{1-e^{-u}}-\frac{1}{u}\right) e^{-u} d u,
$$

it follows that

$$
\left(\frac{\log \widehat{(x+1)}}{x}-\frac{\psi(\widehat{x+1)}+\gamma}{x}\right)(t)=\int_{t}^{+\infty}\left(\frac{1}{1-e^{-u}}-\frac{1}{u}\right) e^{-u} d u
$$

and then one may easily deduce from (29) the relation

$$
\sum_{n \geq 1}^{\mathcal{R}} \frac{\log (n+1)}{n}-\sum_{n \geq 1}^{\mathcal{R}} \frac{H_{n}}{n}=-\frac{\gamma^{2}}{2},
$$

which may be rewritten in the following form (cf. Example 10):

$$
\sum_{n \geq 1}^{\mathcal{R}} \frac{\log (n+1)}{n}=\vartheta(2)-\frac{1}{2} \vartheta(1)^{2} .
$$

\subsection{Link with the Arakawa-Kaneko zeta function}

For $\Re(s) \geq 1$ and $k \geq 1$, one can define in an algebraic fashion the function $\xi_{k}$ by

$$
\xi_{k}(s)=\sum_{n=1}^{\infty} D\left(\left(\frac{1}{x}\right)^{\bowtie k} \bowtie \frac{1}{x^{s}}\right)(n)=\sum_{n=1}^{\infty} \frac{1}{n^{k}} D\left(\frac{1}{x^{s}}\right)(n) .
$$

In particular, for all natural numbers $m$, one has (cf. [8], Corollary 1)

$$
\xi_{k}(m+1)=\sum_{n=1}^{\infty} \frac{1}{n^{k}} D\left(\frac{1}{x^{m+1}}\right)(n)=\sum_{n=1}^{\infty} \frac{P_{m}\left(H_{n}, H_{n}^{(2)}, \ldots, H_{n}^{(m)}\right)}{n^{k+1}} .
$$

Since $D\left(\frac{1}{x^{s}}\right)=\mathcal{L}\left(\frac{\Lambda^{s-1}}{\Gamma(s)}\right)$, one may also rewrite (33) as

$$
\xi_{k}(s)=\frac{1}{\Gamma(s)} \int_{0}^{+\infty} \operatorname{Li}_{k}\left(e^{-u}\right)(\Lambda(u))^{s-1} d u,
$$

and the change of variables $t=\Lambda(u)$ leads to the integral representation

$$
\xi_{k}(s)=\frac{1}{\Gamma(s)} \int_{0}^{+\infty} t^{s-1} \frac{e^{-t}}{1-e^{-t}} \operatorname{Li}_{k}\left(1-e^{-t}\right) d t
$$


which is the analogue of (19) (with $\mathrm{Li}_{k}$ in place of $f_{k}$ ) and also the original definition of the Arakawa-Kaneko zeta function (cf. [1], [8]).

Thus, taking into account the facts that $\xi_{k}(1)=\zeta(k+1)$ and $\operatorname{Li}_{1}\left(1-e^{-t}\right)=t$, and following the same process as in the proof of Theorem 8 , one obtains the following analogue of $(20)$ :

$\xi_{k+1}(s)=\zeta(k+1) \zeta(s)+\sum_{j=1}^{k-1}(-1)^{j} \zeta(k+1-j) Z_{j}(s)+(-1)^{k} \frac{1}{\Gamma(s)} \int_{0}^{+\infty} t^{s-1} \frac{e^{-t}}{1-e^{-t}} T^{k}(t) d t$.

In particular, in the simplest case $k=1$, since

$$
T(t)=\int_{t}^{+\infty} \frac{e^{-u}}{1-e^{-u}} u d u=\sum_{m>0} \int_{t}^{+\infty} e^{-m u} u d u=\sum_{m>0} \frac{e^{-t m}}{m} t+\sum_{m>0} \frac{e^{-t m}}{m^{2}},
$$

(34) again gives the formula

$$
\xi_{2}(s)=\zeta(2) \zeta(s)-s \sum_{n>m>0} \frac{1}{n^{s+1}} \frac{1}{m}-\sum_{n>m>0} \frac{1}{n^{s}} \frac{1}{m^{2}}
$$

already obtained by Arakawa and Kaneko (cf. [1] Theorem 6 (ii)).

\section{Conclusion}

Most of the general results given for the modified zeta function $F_{k}$, especially Theorem 7, Theorem 8, and Theorem 9, also apply (with minor adaptations) to a wide class of functions including the Arakawa-Kaneko zeta function $\xi_{k}$, specifically to the class of functions represented by normalized Mellin transforms of type

$$
F_{k, \omega}(s)=\frac{1}{\Gamma(s)} \int_{0}^{+\infty} t^{s-1} \frac{e^{-t}}{1-e^{-t}} f_{k, \omega}\left(1-e^{-t}\right) d t
$$

with $\omega=\left(\omega_{n}\right)_{n \geq 1}$ and $f_{k, \omega}(z)=\sum_{n=1}^{\infty} \frac{\omega_{n}}{n^{k}} z^{n}$. In particular, under the assumption that $\frac{\left|\omega_{n}\right|}{n^{k}}=O\left(\frac{1}{n}\right)$, we have for positive integers $m$ the nice formula

$$
F_{k, \omega}(m+1)=\sum_{n=1}^{\infty} \frac{\omega_{n}}{n^{k}} D\left(\frac{1}{x^{m+1}}\right)(n)=\sum_{n=1}^{\infty} \omega_{n} \frac{P_{m}\left(H_{n}, H_{n}^{(2)}, \ldots, H_{n}^{(m)}\right)}{n^{k+1}},
$$

which extends (25). However, this formula is more theoretical than practical because of the fast increase in the size of polynomials $P_{m}$ : the number of monomials in $P_{m}$ is equal to the number $p(m)$ of partitions of $m$, as shown by the explicit expression of the $m$ th modified Bell polynomial. 


\section{References}

[1] T. Arakawa and M. Kaneko, Multiple zeta values, Poly-Bernoulli numbers and related zeta functions, Nagoya Math. J. 153 (1999), 189-209.

[2] K. Boyadzhiev, Harmonic number identities via Euler's transform, Journal of Integer Sequences, 12 (2009), Article 09.6.1.

[3] B. Candelpergher, M.A. Coppo, and E. Delabaere, La sommation de Ramanujan, L'Enseignement Mathématique 43 (1997), 93-132.

[4] B.Candelpergher, H. Gadiyar, and R. Padma, Ramanujan summation and the exponential generating function $\sum_{k=0}^{\infty} \frac{z^{k}}{k !} \zeta^{\prime}(-k)$, The Ramanujan J. 21 (2010), 99-122.

[5] X. Chen and W. Chu, Dixon's $F_{2}(1)$-series and identities involving harmonic numbers and the Riemann zeta function, Discrete Math. 310 (2010), 83-91.

[6] J. Choi and H. M. Srivastava, Explicit evaluation of Euler and related sums, The Ramanujan J. 10 (2005), 51-70.

[7] M-A. Coppo, Nouvelles expressions des formules de Hasse et de Hermite pour la fonction zêta d'Hurwitz, Expositiones Math. 27 (2009), 79-86.

[8] M-A. Coppo and B. Candelpergher, The Arakawa-Kaneko Zeta function, The Ramanujan J. 22 (2010), 153-162.

[9] K. Dilcher, Some $q$-series identities related to divisors functions, Discrete Math. 145 (1995), 83-93.

[10] P. Flajolet and R. Sedgewick, Mellin Transforms and Asymptotics: finite differences and Rice's integrals, Theoretical Computer Science 144 (1995), 101-124.

[11] C. Hermite, Extrait de quelques lettres de M. Ch. Hermite à M. S. Pincherle, Annali di Matematica Pura ed Applicata 5 (1901), 55-72.

[12] D. Merlini, R. Sprugnoli, and C. Verri, The Cauchy numbers, Discrete Math. 306 (2006), 1906-1920.

[13] J. Schiff, The Laplace transform : theory and applications, Springer, New-York, 1999.

[14] E. Zeidler, Quantum Field Theory I : Basics in Mathematics and Physics, Springer, Berlin Heidelberg, 2006. 